\title{
Asymptotic behaviour of Betti numbers of real algebraic surfaces
}

F. Bihan

\begin{abstract}
Let $X_{m}$ be a nonsingular real algebraic surface of degree $m$ in the complex projective space $\mathbb{C} P^{3}$ and $\mathbb{R} X_{m}$ its real point set in $\mathbb{R} P^{3}$. In the spirit of the sixteenth Hilbert's problem, one can ask for each degree $m$ about the maximal possible value $\beta_{i, m}$ of the Betti number $b_{i}\left(\mathbb{R} X_{m}\right)$ ( $i=0$ or 1$)$. We show that $\beta_{i, m}$ is asymptotically equivalent to $l_{i} \cdot m^{3}$ for some real number $l_{i}$ and prove inequalities $\frac{13}{36} \leq l_{0} \leq \frac{5}{12}$ and $\frac{13}{18} \leq l_{1} \leq \frac{5}{6}$.
\end{abstract}

Mathematics Subject Classification (2000). 14P25, 14J99.

Keywords. Real algebraic surfaces, Betti numbers, Viro method.

\section{Introduction}

A real algebraic surface $X$ in the complex projective space $\mathbb{C} P^{3}$ is the complex zero set in $\mathbb{C} P^{3}$ of some real homogeneous polynomial in four variables. The real zero set of this polynomial is a surface $\mathbb{R} X$, in the real projective space $\mathbb{R} P^{3}$, called real point set of $X$. (More generally, a real variety is a complex variety $X$ equipped with an antiholomorphic involution $c: X \rightarrow X$, the real point set $\mathbb{R} X$ of $X$ is then the fixed point set of $c$.) From now on $X_{m}$ will denote a real nonsingular surface of degree $m$ in $\mathbb{C} P^{3}$.

This paper deals with the topological classification problem of real point sets $\mathbb{R} X_{m}$ for a given degree $m$ (the case $m=4$ is a part of the sixteen Hilbert's problem going back to 1900) and more specifically with the question of the maximal possible values $\beta_{i, m}\left(i=0\right.$ or 1 ) of the Betti numbers $b_{i}\left(\mathbb{R} X_{m}\right)$ (where homology groups are taken with coefficients in $\mathbb{Z} / 2$ ). The topological classification problem (and thus the last question) is now completely solved for $m \leq 4$ (see [K1]). For $m \geq 5$, even the values of $\beta_{i, m}$ are not known (see [I-K1], [B2] and [O] for the case $m=5$ ). Here, we are interested in the asymptotic behaviour (when $m \rightarrow+\infty$ ) of $\beta_{i, m}$. We first show that $\beta_{i, m}$ is asymptotically equivalent to $l_{i} \cdot m^{3}$ for some real number

The author acknowledges support from the Swiss National Science Foundation. He would like to thank the universities of Geneve and Lausanne for their hospitality. 
$l_{i}$ (Proposition 1.1). A standard application of the Smith-Thom and Comessati inequalities leads to the upper bounds $l_{0} \leq \frac{5}{12}$ and $l_{1} \leq \frac{5}{6}$ (see $[\mathrm{D}-\mathrm{K}]$ for a recent survey on the topology of real algebraic varieties). On the other hand, the best lower bounds previously known were $l_{0} \geq \frac{7}{24}[\mathrm{~V} 2]$ and $l_{1} \geq \frac{97}{144}$ [I2]. In this paper, we shall improve these lower bounds by showing that $l_{0} \geq \frac{13}{36}$ and $l_{1} \geq \frac{13}{18}$ (Theorem 1.2).

We define algebraic surfaces of type $\mathcal{D}_{2 k}$ in some weighted projective space (see 2.1). Such a real nonsingular surface can be equivariantly deformed, by a small deformation, to a real nonsingular surface of degree $2 k$ in $\mathbb{C} P^{3}$ with equation $f_{k}(Z)^{2}-\epsilon \cdot f_{2 k}(Z)=0$, where $f_{i}(Z)$ is a real homogenous polynomial of degree $i$ in $Z=\left(Z_{0}: Z_{1}: Z_{2}: Z_{3}\right)$ and $0<\epsilon \ll 1$ (a small perturbation of a double surface). Assuming that the surfaces $X_{2 k}$ and $X_{k}$ defined by $f_{2 k}$ and $f_{k}$, respectively, are nonsingular surfaces intersecting transversely, the corresponding surface of type $\mathcal{D}_{2 k}$, and thus the real surface in $\mathbb{C} P^{3}$ with equation $f_{k}(Z)^{2}-\epsilon \cdot f_{2 k}(Z)=0$ for small $\epsilon>0$, is then a nonsingular surface whose topological type of real point set is determined by that of the triple $\left(\mathbb{R} P^{3}, \mathbb{R} X_{2 k}, \mathbb{R} X_{k}\right)$. This construction of real nonsingular hypersurfaces resulting from small perturbations of double hypersurfaces is classical in real algebraic geometry and seems to go back, at least, to K. Rohn. In fact, this is essentially the construction used in [V2] where the bound $l_{0} \geq \frac{7}{24}$ is obtained. The deformation we use comes from a paper of E. Horikawa [Ho] where complex surfaces of type $\mathcal{D}_{6}$ are found in the deformation family of sextics in $\mathbb{C} P^{3}$.

In this paper, we apply the above construction in order to produce surfaces in $\mathbb{R} P^{3}$ with large Betti numbers. This is done using the famous construction method of real algebraic hypersurfaces due to O. Viro (see [V3, V4, V5, R]), its combinatorial version the combinatorial patchworking, and the extension of this last version to the case of complete intersections obtained by B. Sturmfels [S1]. The construction presented in this paper has its own interest since it gives an increasing function relating Betti numbers of real algebraic surfaces in $\mathbb{C} P^{3}$ and numbers of even (or odd) ovals of real plane curves (see Remark 2, Section 5). Moreover, this construction can be easily generalized in higher dimensions.

From now on, all polytopes and polyhedral subdivisions shall have integral vertices i.e. vertices with integer coordinates.

\section{Results}

As in the introduction, let $\beta_{i, m}$ denote the maximal possible value of $b_{i}\left(\mathbb{R} X_{m}\right)$ for a given $m(i=0$ or 1$)$. The following result is due to I. Itenberg and V. Kharlamov [I-K2].

Proposition 1.1. The sequence $\beta_{i, m}$ is asymptotically equivalent, when $m \rightarrow$ $+\infty$, to $l_{i} \cdot m^{3}$ for some real number $l_{i}$. 
Proof. By Smith-Thom inequality the sequence $\frac{\beta_{i, m}}{m^{3}}$ is bounded, hence admits a superior limit $l_{i}$. We are going to show that $l_{i}$ is indeed the limit of $\frac{\beta_{i, m}}{m^{3}}$ in the following way: for a given $\epsilon>0$, we construct using the Viro method a family of surfaces $X_{m}$ in $\mathbb{C} P^{3}$ such that $b_{i}\left(\mathbb{R} X_{m}\right) / m^{3} \geq l_{i}-\epsilon$ for any sufficiently large degree $m$.

Let $X_{k}$ be a given surface in $\mathbb{C} P^{3}$ (we shall have $b_{i}\left(\mathbb{R} X_{k}\right) / k^{3}$ close to $l_{i}$ ). For any positive integer number $m$, denote by $T_{m}$ the tetrahedron with vertices $(0,0,0),(m, 0,0),(0, m, 0)$ and $(0,0, m)$ in $\mathbb{R}^{3}$. Let $p$ be any positive integer number. Take any convex triangulation of $T_{p}$ all of whose tetrahedra have euclidian volume $\frac{1}{6}$, or equivalently which contains the maximal number $p^{3}$ of tetrahedra (see 2.2 for the definition of a convex triangulation and [I2] for examples of so-called primitive or unimodular convex triangulations of $T_{p}$ ). Consider now the convex triangulation of the tetrahedron $T_{p(k+4)}$ obtained from the previous triangulation by applying the transformation $x \rightarrow(k+4) \cdot x$. The resulting triangulation contains $p^{3}$ tetrahedra such that each of them has euclidian volume $\frac{(k+4)^{3}}{6}$. The crucial point is that each of these $p^{3}$ tetrahedra contains in its interior the image of $T_{k}$ under some affine unimodular integral transformation. Indeed, any tetrahedron $P$ (with integral vertices) having euclidian volume $\frac{(k+4)^{3}}{6}$ is the image of $T_{k+4}$ under such a transformation $\varphi_{P}$. We have then $\varphi_{P}\left((1,1,1)+T_{k}\right) \subset \operatorname{Int}(P)$ since $(1,1,1)+T_{k} \subset \operatorname{Int}\left(T_{k+4}\right)$. Therefore, we can refine the above triangulation of $T_{p(k+4)}$ in order to obtain a convex triangulation $\left\{Q_{j}, j \in J\right\}$ (here the $Q_{j}$ 's are tetrahedra) containing $p^{3}$ disjoint images of $T_{k}$ by affine unimodular integral transformations. Now, let $\left(Z_{0}: \cdots: Z_{3}\right)$ be homogenous coordinates of $\mathbb{C} P^{3}$ and assume that $\mathbb{R} X_{k} \cap\left\{Z_{i}=0\right\}=\emptyset$ for $i=0, \cdots, 3$. Let $f$ be some affine polynomial defining the surface $X_{k}$ in the chart $\left\{Z_{0} \neq 0\right\}$ with affine coordinates $\left(\frac{Z_{1}}{Z_{0}}, \frac{Z_{2}}{Z_{0}}, \frac{Z_{3}}{Z_{0}}\right)$. The previous assumption implies that $\mathbb{R} X_{k}=\{f=0\} \cap\left(\mathbb{R}^{*}\right)^{3}$. We can associate to the triangulation $\left\{Q_{j}, j \in J\right\}$ of $T_{p(k+4)}$ a collection $\left\{f_{j}, j \in J\right\}$ of nondegenerate polynomials (see 2.2 for the definition of a nondegenerate polynomial) verifying the following conditions:

- $f_{j}$ has $Q_{j}$ as Newton polytope for any $j \in J$,

- the truncation of $f_{j}$ and $f_{j^{\prime}}$ to a common face of $Q_{j}$ and $Q_{j^{\prime}}$ coincide,

- if $Q_{j}$ is one of the $p^{3}$ images of $T_{k}$ under some affine unimodular integral transformation, then $f_{j}$ is the image of $f$ under the corresponding monomial change of coordinates.

The first two conditions allow us to apply the Viro method in order to construct a nonsingular surface $X_{p(k+4)}$ whose real point set is homeomorphic to a topological surface obtained by gluing together the sets $\left\{f_{j}=0\right\} \cap\left(\mathbb{R}^{*}\right)^{3}$ for $j \in J$ (the gluing being determined by the triangulation). The third condition ensures that $\left\{f_{j}=0\right\} \cap\left(\mathbb{R}^{*}\right)^{3}$ is homeomorphic to $\{f=0\} \cap\left(\mathbb{R}^{*}\right)^{3}$, hence to $\mathbb{R} X_{k}$. It follows that these $p^{3}$ homeomorphic copies of $\mathbb{R} X_{k}$ are disjoint in $\mathbb{R} X_{p(k+4)}$ since the gluing is made along intersections of the $\left\{f_{j}=0\right\}$ with the coordinate and infinity planes. Note also that for the same reason these $p^{3}$ homeomorphic copies of $\mathbb{R} X_{k}$ contained 
in $\mathbb{R} X_{p(k+4)}$ do not intersect the coordinate or infinity planes. In particular, we obtain $b_{i}\left(\mathbb{R} X_{p(k+4)}\right) \geq p^{3} \cdot b_{i}\left(\mathbb{R} X_{k}\right)$, which leads to

$$
\frac{b_{i}\left(\mathbb{R} X_{p(k+4)}\right)}{p^{3} \cdot(k+4)^{3}} \geq \frac{b_{i}\left(\mathbb{R} X_{k}\right)}{k^{3}}-\frac{b_{i}\left(\mathbb{R} X_{k}\right)}{k^{3}} \cdot\left[1-\frac{1}{(1+4 / k)^{3}}\right] .
$$

Let $\epsilon>0$ be given. From the definition of $l_{i}$, for sufficiently large $k$ there exists a surface $X_{k}$ verifying

$$
\frac{b_{i}\left(\mathbb{R} X_{k}\right)}{k^{3}} \geq l_{i}-\epsilon / 2 .
$$

Then, such a surface can obviously be chosen in order to verify $\mathbb{R} X_{k} \cap\left\{Z_{i}=0\right\}=\emptyset$ for $i=0, \cdots, 3$. Recall that the sequence $\frac{\beta_{i, m}}{m^{3}}$ is bounded. Therefore, by (1) one can also assume that the surface $X_{p(k+4)}$ constructed from $X_{k}$ verifies

$$
\frac{b_{i}\left(\mathbb{R} X_{p(k+4)}\right)}{p^{3} \cdot(k+4)^{3}} \geq \frac{b_{i}\left(\mathbb{R} X_{k}\right)}{k^{3}}-\epsilon / 4 \text {. }
$$

Now, fix some sufficiently large $k$ in order to have the inequalities (2) and (3) and consider a family of surfaces $\left\{X_{p(k+4)}, p \geq p_{0}\right\}$ constructed as before for each $p \geq p_{0}$, where $p_{0}$ is some positive integer number. Extend this family to a whole family $\mathcal{F}=\left\{X_{m}, m \geq p_{0}(k+4)\right\}$ in the following way: if $m$ is any integer number such that $p(k+4)<m<(p+1)(k+4)$ for some integer $p \geq p_{0}$, then consider the union of $X_{p(k+4)}$ with $m-p(k+4)$ planes which do not intersect the $p^{3}$ homeomorphic copies of $\mathbb{R} X_{k}$ contained in $\mathbb{R} X_{p(k+4)}$ (as we have noticed before, these $p^{3}$ homeomorphic copies do not intersect the coordinate planes, hence we can take planes which are close to the coordinate ones) and take for $X_{m}$ any smoothing of the resulting surface. It follows that $b_{i}\left(\mathbb{R} X_{m}\right) \geq p^{3} \cdot b_{i}\left(\mathbb{R} X_{k}\right)$ and, using $m<(p+1)(k+4)$, we obtain

$$
\frac{b_{i}\left(\mathbb{R} X_{m}\right)}{m^{3}} \geq \frac{b_{i}\left(\mathbb{R} X_{k}\right)}{k^{3}}-\frac{b_{i}\left(\mathbb{R} X_{k}\right)}{k^{3}} \cdot\left[1-\frac{1}{[(1+4 / k)(1+1 / p)]^{3}}\right] .
$$

Recall that the sequence $\frac{\beta_{i, m}}{m^{3}}$ is bounded. Consequently, if $p_{0}$ is chosen sufficiently large, then by (4) and (3), for any surface $X_{m} \in \mathcal{F}$ we shall have

$$
\frac{b_{i}\left(\mathbb{R} X_{m}\right)}{m^{3}} \geq \frac{b_{i}\left(\mathbb{R} X_{k}\right)}{k^{3}}-\epsilon / 2 .
$$

and thus by $(2)$

$$
\frac{b_{i}\left(\mathbb{R} X_{m}\right)}{m^{3}} \geq l_{i}-\epsilon
$$

Remark 1.1. The previous proof works in any dimension. Namely, one can show the asymptotic equivalence, when $m \rightarrow+\infty$, between the sequence $\beta_{i, m}^{(n)}$ of the maximal possible values of the Betti numbers $b_{i}\left(\mathbb{R} X_{m}^{n}\right)(i=0, \cdots, n-1)$ and some real number $l_{i}^{(n)}$ times $m^{n}$ (where $i, n$ are fixed and $X_{m}^{n}$ denotes a nonsingular real algebraic hypersurface of degree $m$ in $\left.\mathbb{C} P^{n}\right)$. 
The largest part of the paper is devoted to constructions of surfaces proving the following result.

Theorem 1.1. Let $i=0$ or 1 . For any $\epsilon>0$, there exists a family of surfaces $X_{m}$ in $\mathbb{C} P^{3}$ such that

$$
b_{i}\left(\mathbb{R} X_{m}\right)>\left(\frac{13(1+i)}{36}-\epsilon\right) \cdot m^{3}
$$

for any sufficiently large degree $m$.

As a corollary of Theorem 1.1 and the classical upper bounds $l_{0} \leq \frac{5}{12}, l_{1} \leq \frac{5}{6}$, we obtain the main result of this paper.

Theorem 1.2. The real numbers $l_{0}$ and $l_{1}$ verify

$$
\frac{13}{36} \leq l_{0} \leq \frac{5}{12} \quad \text { and } \quad \frac{13}{18} \leq l_{1} \leq \frac{5}{6}
$$

\section{Constructions}

\subsection{Surfaces of type $\mathcal{D}_{2 k}$}

Denote by $\mathbb{C} P^{4}(k)$ the four dimensional weighted complex projective space with homogeneous coordinates $Z_{0}, Z_{1}, Z_{2}, Z_{3}$ of weight 1 and $Z_{4}$ of weight $k$ (where $k$ is some positive integer number). A real surface of type $\mathcal{D}_{2 k}$ is a surface $Y$ defined in $\mathbb{C} P^{4}(k)$ by a system of equations of type

$$
\left\{\begin{array}{l}
Z_{4}{ }^{2}-f_{2 k}(Z)=0, \\
f_{k}(Z)=0
\end{array}\right.
$$

where $f_{2 k}(Z)$ and $f_{k}(Z)$ are real homogeneous polynomials of degree $2 k$ and $k$ in the variables $Z=\left(Z_{0}: Z_{1}: Z_{2}: Z_{3}\right)$, respectively. The surface $Y$ is then real with respect to the standard complex conjugation $\left(Z: Z_{4}\right) \rightarrow\left(\bar{Z}: \bar{Z}_{4}\right)$ in $\mathbb{C} P^{4}(k)$. Assume that the real surfaces $X_{2 k}$ and $X_{k}$ defined, respectively, by $f_{2 k}$ and $f_{k}$ in $\mathbb{C} P^{3}$ are nonsingular surfaces (this will be always the case later). Then, we shall say that $Y$ is associated with the pair $\left(X_{2 k}, X_{k}\right)$.

Proposition 2.1 (see [Ho]). Let $Y$ be a real surface of type $\mathcal{D}_{2 k}$ associated with a pair of (nonsingular) surfaces $\left(X_{2 k}, X_{k}\right)$ whose real point sets intersect transversely. Then, there exists a small equivariant deformation of $Y$ to a real nonsingular algebraic surface of degree $2 k$ in $\mathbb{C} P^{3}$. In particular, the real point set of the latter surface is homeomorphic to $\mathbb{R} Y$.

Proof. A desired deformation is given by the family of surfaces $Y^{\epsilon}, \epsilon \in \mathbb{R}$ and $Y^{0}=Y$, defined in $\mathbb{C} P^{4}(k)$ by $\left\{Z_{4}{ }^{2}-f_{2 k}(Z)=0, f_{k}(Z)-\epsilon Z_{4}=0\right\}$. The 
projection $\mathbb{C} P^{4}(k) \backslash\{(0: 1)\} \rightarrow \mathbb{C} P^{3},\left(Z: Z_{4}\right) \rightarrow Z$, produces an equivariant isomorphism between $Y^{\epsilon}, \epsilon \neq 0$, and the real surface $X^{\epsilon}$ in $\mathbb{C} P^{3}$ defined by $\left(\frac{f_{k}(Z)}{\epsilon}\right)^{2}-f_{2 k}(Z)$. Since $\mathbb{R} X_{2 k}$ and $\mathbb{R} X_{k}$ are nonsingular surfaces intersecting transversely, both surfaces $\mathbb{R} Y$ and $\mathbb{R} X^{\epsilon}$ are nonsingular and homeomorphic for sufficiently small $\epsilon$.

Let $Y$ be a surface of type $\mathcal{D}_{2 k}$ as in Proposition 2.1, then $Y$ is isomorphic to the double covering of $X_{k}$ branched along the curve $X_{k} \cap X_{2 k}$. Moreover, the topology of $\mathbb{R} Y$ is given by that of the triple $\left(\mathbb{R} P^{3}, \mathbb{R} X_{2 k}, \mathbb{R} X_{k}\right)$. Namely, the surface $\mathbb{R} Y$ is projected two-to-one onto the interior of $\mathbb{R} X_{k}^{+}=\mathbb{R} X_{k} \cap\left\{f_{2 k}(Z) \geq 0\right\}$ and one-to-one onto the boundary $\mathbb{R} X_{2 k} \cap \mathbb{R} X_{k}$ of $\mathbb{R} X_{k}^{+}$.

$$
\stackrel{\mathbb{R} Y}{\stackrel{\perp}{\perp} X_{2 k} \cap \mathbb{R} X_{k} \subset \mathbb{R} X_{k}^{+}}
$$

\subsection{A particular case of Sturmfels's theorem}

We use the extension due to Sturmfels [S1] of the combinatorial patchworking in order to construct a pair of surfaces $\left(X_{2 k}, \widehat{X}_{k}\right)$, where $X_{2 k}$ is a real nonsingular surface of degree $2 k$ in $\mathbb{C} P^{3}$ and $\widehat{X}_{k}$ is a real surface of degree $k$ in $\mathbb{C} P^{3}$ having a single nondegenerate point as singularities. In the rest of this paper, we shall assume $k \geq 3$. Consider the sets

$$
\begin{aligned}
& \mathcal{P}_{1}=\left\{(x, y, z) \in \mathbb{Z}^{3}, x, y, z \geq 0, k-1 \leq x+y+z \leq k\right\} \\
& \mathcal{P}_{2}=\left\{(x, y, z) \in \mathbb{Z}^{3}, x, y, z \geq 0, x+y+z \leq 2 k\right\}
\end{aligned}
$$

and denote by $P_{i}$ the convex hull of $\mathcal{P}_{i}$. For each positive real number $t$, let $f_{i, t}(z) \in \mathbb{R}\left[z_{1}, z_{2}, z_{3}\right]$ be a so-called affine Viro polynomial defined by

$$
f_{i, t}(z)=\sum_{w \in \mathcal{P}_{i}} s_{i}(w) t^{\nu_{i}(w)} z^{w}
$$

where $s_{i}$ takes values $\pm 1\left(s_{i}(w)\right.$ will be sometimes called sign of $\left.w\right)$ and $\nu_{i}: P_{i} \rightarrow$ $\mathbb{R}_{+}$is a convex function. Define the polytope $P^{\nu_{i}}=\operatorname{conv}\left\{\left(w, \nu_{i}(w)\right), w \in \mathcal{P}_{i}\right\}$ and denote by $G^{\nu_{i}}$ the lower part of $P^{\nu_{i}}$. By projecting faces of $G^{\nu_{i}} \subset \mathbb{R}^{3} \times \mathbb{R}$ onto $\mathbb{R}^{3}$, the function $\nu_{i}$ defines a polyhedral subdivision $\tau_{i}$ of $P_{i}$. Polyhedral subdivisions of polytopes obtained in this way are called convex. Assume that $\tau_{1}$ and $\tau_{2}$ are triangulations with sets of vertices $\mathcal{P}_{1}$ and $\mathcal{P}_{2}$, respectively. In this case, the above Viro polynomials are called $T$-polynomials.

Consider the Minkowsky sums $P=P_{1}+P_{2}, P^{\nu}=P^{\nu_{1}}+P^{\nu_{2}}$ and denote by $G^{\nu}$ the lower part of $P^{\nu}$. Projecting faces of $G^{\nu} \subset \mathbb{R}^{3} \times \mathbb{R}$ onto $\mathbb{R}^{3}$, we get a polyhedral subdivision $\tau$ of $P$. Each face $F^{\nu}$ of $G^{\nu}$ has a unique representation $F^{\nu}=F^{\nu_{1}}+F^{\nu_{2}}$, where $F^{\nu_{i}}$ is a face of $G^{\nu_{i}}$, which induces, via the projection, a particular representation $F=F_{1}+F_{2}$, where $F_{i}$ is a simplex of $\tau_{i}$, of each 
polytope $F$ of the subdivision $\tau$. We shall refer to this particular representation when writing $F=F_{1}+F_{2}$ with $F \in \tau$ and $F_{i} \in \tau_{i}$. Assume that for each polytope $F=F_{1}+F_{2}$ of $\tau$, we have $\operatorname{dim}(F)=\operatorname{dim}\left(F_{1}\right)+\operatorname{dim}\left(F_{2}\right)$. Then, the subdivision (or decomposition) $\tau$ is called mixed decomposition ([S1]).

Let $\mathcal{R}^{n}$ be the group of symmetries in $\mathbb{R}^{n}$ generated by reflections with respect to the coordinate hyperplanes. Let us identify $(\mathbb{Z} / 2)^{n}$ with $\mathcal{R}^{n}$ by sending $r=$ $\left(r_{1}, \cdots, r_{n}\right)$ to $\left(\left(x_{1}, \cdots, x_{n}\right) \rightarrow\left((-1)^{r_{1}} x_{1}, \cdots,(-1)^{r_{1}} x_{n}\right)\right)$. For any polytope $T$ in $\mathbb{R}^{n}$, let $T^{*}$ be the union of all the symmetric copies $r(T)$ of $T$ for $r \in \mathcal{R}^{n}$. For any face $\Gamma$ of $T^{*}$ and any vector $\alpha \in \mathbb{Z}^{n}$ orthogonal to $\Gamma$, identify $\Gamma$ with its symmetric copy $\bar{\alpha}(\Gamma)$ (where $\bar{\alpha}$ denote the reduction of $\alpha$ in $\left.(\mathbb{Z} / 2)^{n}\right)$. Denote by $\tilde{T}$ the resulting space.

Denote by $\tau^{*}$ (resp. $\tau_{i}^{*}$ ) the unique subdivision of $P^{*}$ (resp. $P_{i}^{*}$ ) which extends $\tau$ (resp. $\tau_{i}$ ) and which is invariant with respect to $\mathcal{R}^{n}$. Extend $s_{i}$ to a distribution of signs on the set of vertices of $\tau_{i}^{*}$ following the rule: $s_{i}(r(w))=s_{i}(w) \cdot(-1)^{\langle r, \bar{w}\rangle}$, where $\langle$,$\rangle is the usual scalar product in (\mathbb{Z} / 2)^{3}$. For each vertex $w=w_{1}+w_{2}$ of $\tau^{*}$, where $w_{i} \in \tau_{i}^{*}$, define its sign vector $s(w)=\left(s_{1}\left(w_{1}\right), s_{2}\left(w_{2}\right)\right)$.

We perform the construction of piecewise linear surfaces in $\tilde{P}_{i}$ and $\tilde{P}$. For any tetrahedron of $\tau_{i}^{*}$ having at least two vertices with different signs, consider its edges having endpoints with different signs and select the triangle or quadrangle having the middle points of these edges as vertices. The union of all the selected pieces is a piecewise linear surface in $P_{i}^{*}$. Denote by $\tilde{S}_{i}$ its image in $\tilde{P}_{i}$. For any three-dimensional polytope (a prism or a tetrahedron) $F=F_{1}+F_{2}$ of $\tau^{*}$ such that $\tilde{S}_{1} \cap F_{1} \neq \emptyset$, select the Minkowsky sum $\left(\tilde{S}_{1} \cap F_{1}\right)+F_{2}$ (a quadrangle or a triangle). The union of all the selected pieces is a piecewise linear surface in $P^{*}$. Denote by $\tilde{S}^{1}$ its image in $\tilde{P}$. Similarly, for any three-dimensional polytope $F=F_{1}+F_{2}$ of $\tau^{*}$ such that $\tilde{S}_{2} \cap F_{2} \neq \emptyset$, select $F_{1}+\left(\tilde{S}_{2} \cap F_{2}\right)$. The union of all the selected pieces is a piecewise linear surface in $P^{*}$ whose image in $\tilde{P}$ is denoted by $\tilde{S}^{2}$.

Remark 2.1. By construction, we clearly have the following properties.

(1) The distribution $s_{i}$ is constant on each connected component of $\tilde{P}_{i} \backslash \tilde{S}_{i}$ and its value changes while passing through $\tilde{S}_{i}$.

(2) The coordinate $s_{i}$ of the distribution of sign vectors $s$ is constant on each connected component of $\tilde{P} \backslash \tilde{S}^{i}$ and its value changes while passing through $\tilde{S}^{i}$.

Consider the affine chart $\left\{Z_{0} \neq 0\right\}$ of $\mathbb{C} P^{3}$ with coordinates $z=\left(z_{1}, z_{2}, z_{3}\right)$, where $z_{i}=\frac{Z_{i}}{Z_{0}}$. Let $q: \mathbb{C} \tilde{P}^{3} \rightarrow \mathbb{C} P^{3}$ be the blowing up of $\mathbb{C} P^{3}$ at the point $O=(1: 0: 0: 0)$ (so $O$ has affine coordinates $(0,0,0)$ in $\left\{Z_{0} \neq 0\right\}$ ). Consider the real structure on $\mathbb{C} \tilde{P}^{3}$ which lifts that of $\mathbb{C} P^{3}$. Let $\widehat{X}_{k}$ and $X_{2 k}$ be the real surfaces in $\mathbb{C} P^{3}$ which are defined in the chart $\left\{Z_{0} \neq 0\right\}$ by $f_{1, t}$ and $f_{2, t}$, respectively. Denote by $L$ the exceptional divisor of $q$ and by $W_{1}, W_{2}$ the strict transforms of $\widehat{X}_{k}$ and $X_{2 k}$, respectively. Finally, denote by $p$ the facet of $P$ supported by the plane $\{x+y+z=k-1\}$. 
The following proposition is a particular case of a result of Sturmfels.

Proposition 2.2. Assume that $\tau_{i}(i=1,2)$ is a triangulation with set of vertices $\mathcal{P}_{i}$ and $\tau$ is a mixed decomposition of $P$. Then, for sufficiently small $t>0$, the polynomials $f_{1, t}$ and $f_{2, t}$ are nondegenerate, the surfaces $\mathbb{R} W_{1}$ and $\mathbb{R} W_{2}$ intersect transversely, and there exists a homeomorphism $h: \mathbb{R} \tilde{P}^{3} \rightarrow \tilde{P}$ sending $\mathbb{R} L, \mathbb{R} W_{1}$ and $\mathbb{R} W_{2}$ to $\tilde{p}, \tilde{S}^{1}$ and $\tilde{S}^{2}$, respectively. Consequently, if $\mathcal{U}$ is any neighbourhood of $O \in \mathbb{R} P^{3}$ and $\mathcal{V}=h \circ q^{-1}(\mathcal{U})$, then $h \circ q^{-1}$ induces a homeomorphism $\mathbb{R} P^{3} \backslash \mathcal{U} \rightarrow$ $\tilde{P} \backslash \mathcal{V}$ sending $\mathbb{R} \widehat{X}_{k} \backslash \mathbb{R} \widehat{X}_{k} \cap \mathcal{U}$ and $\mathbb{R} X_{2 k} \backslash \mathbb{R} X_{2 k} \cap \mathcal{U}$ to $\tilde{S}^{1} \backslash \tilde{S}^{1} \cap \mathcal{V}$ and $\tilde{S}^{2} \backslash \tilde{S}^{2} \cap \mathcal{V}$, respectively.

An affine polynomial $f$ in $n$ variables is nondegenerate if for any face $\Gamma$ of its Newton polytope (including the Newton polytope itself), the truncation $f^{\Gamma}$ of $f$ to $\Gamma$ defines a nonsingular hypersurface in the real torus $\left(\mathbb{R}^{*}\right)^{n}$ (see $[\mathrm{R}]$ ). The non-degeneracy of $f_{1, t}$ and $f_{2, t}$ (for sufficiently small $t>0$ ) implies that $\mathbb{R} W_{1}$, $\mathbb{R} W_{2}, \mathbb{R} X_{2 k}$ are nonsingular surfaces and the surface $\mathbb{R} \widehat{X}_{k}$ has the single point $O$ as singularities (moreover $O$ is a nondegenerate singularity of $\mathbb{R} \widehat{X}_{k}$ ). Furthermore, we obtain that for sufficiently small $t>0$ the surfaces $\mathbb{R} \widehat{X}_{k}$ and $\mathbb{R} X_{2 k}$ intersect transversely since, by Proposition 2.2 , this is the case for the surfaces $\mathbb{R} W_{1}$ and $\mathbb{R} W_{2}$.

\subsection{Smoothing}

Let $\widehat{X}_{k}$ be a given real surface in $\mathbb{C} P^{3}$ defined by some nondegenerate polynomial $f_{1}$ having as Newton polytope the polytope $P_{1}$ described in 2.2 . We use the Viro method in order to smooth $\widehat{X}_{k}$.

For any positive integer number $m$, let us denote by $T_{m}$ the tetrahedron which is the convex hull of $\{(x, y, z), x, y, z \geq 0, x+y+z \leq m\}$. Decompose the tetrahedron $T_{k}$ into the union of the polytopes $P_{1}, T_{k-2}$ and the intermediate polytope $I=\operatorname{conv}\{(x, y, z), x, y, z \geq 0, k-2 \leq x+y+z \leq k-1\}$. Let $v$ and $g$ be nondegenerate real polynomials with Newton polytopes $T_{k-2}$ and $I$, respectively, and assume that any truncation of two polynomials among $f_{1}, v$ and $g$ to a common face of their Newton polytopes coincide. Consider any convex function $\nu: T_{k} \rightarrow \mathbb{R}_{+}$which is linear on $P_{1}, T_{k-2}$ and $I$ but not linear on the union of any two of them, and which vanishes identically on $P_{1}$ but is strictly positive outside $P_{1}$. For example, take $\nu(x, y, z)=0$ on $P_{1}, \nu(x, y, z)=(k-1)-(x+y+z)$ on $I$ and $\nu(x, y, z)=2 k-3-2(x+y+z)$ on $T_{k-2}$. The associated Viro polynomial $p_{t}(z)$ is equal to $f_{1}(z)$ plus a polynomial with the property that each of its coefficients contains $t$ to a strictly positive real number. Therefore, the polynomial $p_{t}(z)$ defines for a sufficiently small $t$ a real surface $X_{k}$ of degree $k$ in $\mathbb{C} P^{3}$ which is a small perturbation of $\widehat{X}_{k}$. By Viro theorem (see [V3] or Theorem 4.2 in [R]) we obtain that $\mathbb{R} X_{k}$ is a nonsingular surface homeomorphic to a topological surface 
obtained by gluing together $\left\{f_{1}=0\right\} \cap\left(\mathbb{R}^{*}\right)^{3},\{v=0\} \cap\left(\mathbb{R}^{*}\right)^{3}$ and $\{g=0\} \cap\left(\mathbb{R}^{*}\right)^{3}$ following the subdivision of $T_{k}$. In particular, if $V_{k-2}$ denotes the nonsingular real surface of degree $k-2$ in $\mathbb{C} P^{3}$ defined by the polynomial $v$, the Viro theorem implies the following statement.

Proposition 2.3. For sufficiently small $t>0$, the polynomial $p_{t}(z)$ is nondegenerate and there exist two small neighbourhoods $\mathcal{U}_{1}$ and $\mathcal{U}_{2}$ of $O \in \mathbb{R} P^{3}, \mathcal{U}_{1} \subset \mathcal{U}_{2}$, which can be chosen as small as wanted for $t$ small enough, such that $\mathbb{R} X_{k}$ is homeomorphic,

(1) inside $\mathcal{U}_{1}$, to some affine part of $\mathbb{R} V_{k-2}$,

(2) outside $\mathcal{U}_{2}$, to $\mathbb{R} \widehat{X}_{k}$ outside a small neighbourhood of $O \in \mathbb{R} P^{3}$.

Furthermore, we have

$$
b_{i}\left(\mathbb{R} X_{k}\right)=b_{i}\left(\mathbb{R} V_{k-2}\right)+O\left(k^{2}\right) .
$$

The last inequality in Proposition 2.3 is an easy consequence of the SmithThom inequality together with the equality $6\left[\operatorname{vol}\left(T_{k}\right)-\operatorname{vol}\left(T_{k-2}\right)\right]=k^{3}-(k-2)^{3}=$ $O\left(k^{2}\right)$ (where $\operatorname{vol}(\cdot)$ denotes the euclidian volume). This inequality will be useful later since we shall be interested in the Betti numbers of $\mathbb{R} X_{k}$ for $k$ big enough.

\subsection{Real surfaces of type $\mathcal{D}_{2 k}$}

Let $X_{2 k}$ and $\widehat{X}_{k}$ be surfaces in $\mathbb{C} P^{3}$ defined by T-polynomials $f_{2, t_{0}}$ and $f_{1, t_{0}}$, respectively, as in 2.2 , and assume that the value $t_{0}$ is sufficiently small in order to have the properties stated in Proposition 2.2. Perform the smoothing of $\widehat{X}_{k}$ described in 2.3 and let $X_{k}$ be the resulting surface defined by the Viro polynomial $p_{t}$. For $t>0$ sufficiently small, the surface $X_{k}$ is a nonsingular surface, furthermore, its real point set $\mathbb{R} X_{k}$ intersects $\mathbb{R} X_{2 k}$ transversely, because so does $\mathbb{R} \widehat{X}_{k}$ and $X_{k}$ is a small deformation of $\widehat{X}_{k}$. Consequently, if $Y$ is the surface of type $\mathcal{D}_{2 k}$ associated with $\left(X_{2 k}, X_{k}\right)$, then $\mathbb{R} Y$ is nonsingular and there exists (Proposition 2.1) a small deformation of $Y$ to a real nonsingular surface of degree $2 k$ in $\mathbb{C} P^{3}$ whose real point set is homeomorphic to $\mathbb{R} Y$.

The topogical type of $\mathbb{R} Y$ (in fact, its "asymptotic part") will be obtained using the proposition below. Recall that the topological type of $\mathbb{R} Y$ is closely related to that of $\mathbb{R} X_{k}^{+}=\mathbb{R} X_{k} \cap\left\{f_{2, t_{0}}(Z) \geq 0\right\}$.

Proposition 2.4. Let $\mathcal{U}$ be any neighbourhood of $O$ in $\mathbb{R} P^{3}$ and $\mathcal{V}=h \circ q^{-1}(\mathcal{U})$ be the corresponding neighbourhood of $\tilde{p}$ in $\tilde{P}$ (see Proposition 2.2). Suppose $t>0$ is sufficiently small.

Then, there exists a homeomorphism

$$
\begin{gathered}
\mathbb{R} P^{3} \backslash \mathcal{U} \longrightarrow \tilde{P} \backslash \mathcal{V} \\
\text { sending } \mathbb{R} X_{k} \backslash \mathbb{R} X_{k} \cap \mathcal{U} \text { to } \tilde{S}^{1} \backslash \tilde{S}^{1} \cap \mathcal{V} \text { and } \mathbb{R} X_{2 k} \backslash \mathbb{R} X_{2 k} \cap \mathcal{U} \text { to } \tilde{S}^{2} \backslash \tilde{S}^{2} \cap \mathcal{V} .
\end{gathered}
$$


Moreover, if $\mathcal{U} \cap \mathbb{R} X_{2 k}=\emptyset$ and $s_{2}((0,0,0))=+1$ (the sign given by $s_{2}$ at the vertex $(0,0,0)$ of $\left.P_{2}\right)$, then

- $\mathbb{R} X_{k}^{+} \cap \mathcal{U}$ coincides with $\mathbb{R} X_{k} \cap \mathcal{U}$,

- the above homeomorphism $\mathbb{R} P^{3} \backslash \mathcal{U} \rightarrow \tilde{P} \backslash \mathcal{V}$ sends $\mathbb{R} X_{k}^{+} \cap\left(\mathbb{R} P^{3} \backslash \mathcal{U}\right)$ to $\tilde{S}^{1,+} \cap$ $(\tilde{P} \backslash \mathcal{V})$, where $\tilde{S}^{1,+}$ is the intersection of $\tilde{S}^{1}$ with the connected components of $\widetilde{P} \backslash \tilde{S}^{2}$ containing vertices on which $s_{2}$ takes the value +1 .

Proof. For sufficiently small values of $t$, the surface $\mathbb{R} X_{k}$ is homeomorphic to $\mathbb{R} \widehat{X}_{k}$ in the complementary part of $\mathcal{U}$ in $\mathbb{R} P^{3}$, and intersects transversely $\mathbb{R} X_{2 k}$. Hence, for sufficiently small $t$, the pair $\left(\mathbb{R} X_{2 k}, \mathbb{R} X_{k}\right)$ is homeomorphic to $\left(\mathbb{R} X_{2 k}, \mathbb{R} \widehat{X}_{k}\right)$ in the complementary part of $\mathcal{U}$ in $\mathbb{R} P^{3}$. It remains to use Proposition 2.2 to obtain the desired homeomorphism $\mathbb{R} P^{3} \backslash \mathcal{U} \rightarrow \tilde{P} \backslash \mathcal{V}$.

Suppose now $\mathcal{U} \cap \mathbb{R} X_{2 k}=\emptyset$ and $s_{2}((0,0,0))=+1$. Then $f_{2, t_{0}}$ takes a positive value in the point $O$, hence in the whole $\mathcal{U}$ since $\mathcal{U} \cap \mathbb{R} X_{2 k}=\emptyset$. Consequently, connected components of $\mathbb{R} X_{k}$ contained in $\mathcal{U}$ are connected components of $\mathbb{R} X_{k}^{+}$. Moreover, the distribution $s_{2}$ takes the value +1 in all the vertices of $\tau^{*}$ contained in $p^{*}$, hence in $\mathcal{V}$ since $\mathcal{V} \cap \tilde{S}^{2}=\emptyset$. The last part of the proposition is now obvious in view of Remark 2.1.

\section{Application}

We apply simultaneously the construction described in Section 2 in order to obtain two real nonsingular surfaces $Y_{2 k}^{c}$ and $Y_{2 k}^{h}$ of type $\mathcal{D}_{2 k}$ associated with pairs $\left(X_{2 k}^{c}, X_{k}\right)$ and $\left(X_{2 k}^{h}, X_{k}\right)$, respectively. The surfaces $Y_{2 k}^{c}$ will give, via deformation, surfaces in $\mathbb{R} P^{3}$ with a large number of connected components while the surfaces $Y_{2 k}^{h}$ will give surfaces in $\mathbb{R} P^{3}$ with a large number of handles (superscripts "c" and "h" are for components and handles, respectively).

Let us describe a mixed decomposition $\tau$ of $P$ and distributions of sign vectors $\left(s_{1}, s_{2}^{c}\right)$ and $\left(s_{1}, s_{2}^{h}\right)$ producing as in 2.2 pairs of surfaces $\left(\widehat{X}_{k}, X_{2 k}^{c}\right)$ and $\left(\widehat{X}_{k}, X_{2 k}^{h}\right)$, respectively. Denote by $p_{1}$ the facet of $P_{1}$ supported by the plane $\{x+y+z=k\}$ and by $p_{2}$ the facet of $P_{2}$ supported by $\{z=0\}$. The decomposition $\tau$ of $P$ we are going to use is obtained as follows. First, decompose $P$ into the Minkowsky sums (see Figure 1)

$$
\begin{array}{ll}
P_{1}+(0,0,0), & \Lambda_{1}=p_{1}+[(0,0,0),(2 k, 0,0)], \\
(0,0, k)+P_{2}, & \Lambda_{2}=[(0,0, k),(0, k, 0)]+p_{2} .
\end{array}
$$

Then, subdivise all these polytopes using convex triangulations $\tau_{1}$ and $\tau_{2}$ of $P_{1}$ and $P_{2}$, respectively. Namely, the decomposition consists of the following three dimensional polytopes:

- $\sigma+(0,0,0)$ for any tetrahedron $\sigma \in \tau_{1}$,

- $(0,0, k)+\sigma$ for any tetrahedron $\sigma \in \tau_{2}$,

- $\sigma_{1}+\sigma_{2}$ for any triangle $\sigma_{1} \in \tau_{1}$ contained in $p_{1}$ and any edge $\sigma_{2} \in \tau_{2}$ contained in the edge $[(0,0,0),(2 k, 0,0)]$ of $P_{2}$, 
- $\sigma_{1}+\sigma_{2}$ for any edge $\sigma_{1} \in \tau_{1}$ contained in the edge $[(0,0, k),(0, k, 0)]$ of $P_{1}$ and any triangle $\sigma_{2} \in \tau_{1}$ contained in $p_{2}$.

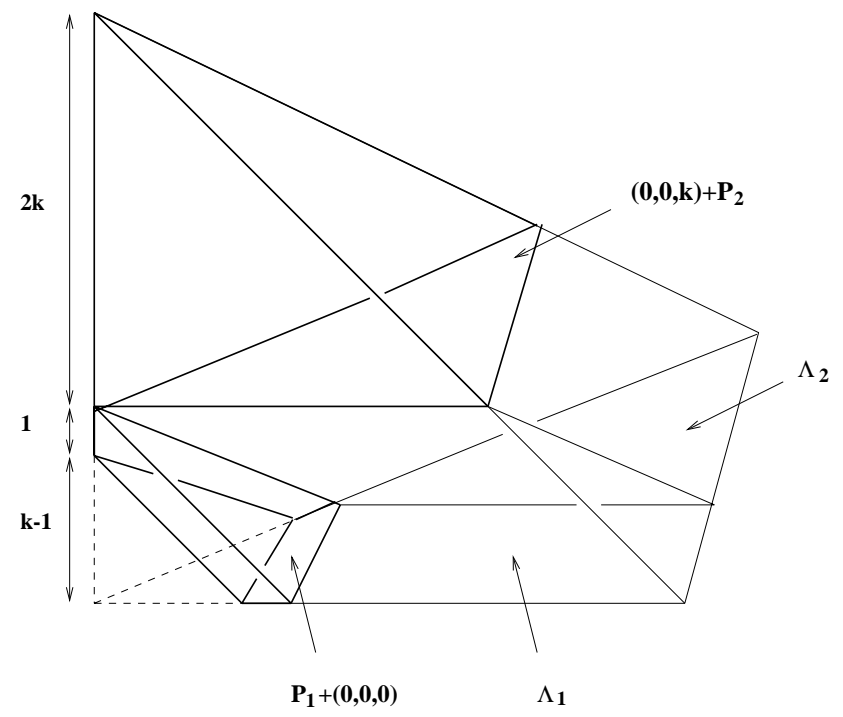

FIG. 1. A mixed decomposition of $P$

Let $Q \subset \mathbb{R} \times \mathbb{R}^{3}$ be the convex hull of the set $\left\{e_{1}\right\} \times \mathcal{P}_{1} \cup\left\{e_{2}\right\} \times \mathcal{P}_{2}$ (where $e_{i}$ is an integer number). In other words, the polytope $Q$ is the join $\left\{e_{1}\right\} \times P_{1} \star\left\{e_{2}\right\} \times P_{2}$.

Lemma 3.1 (see [S2]). Mixed decompositions of $P$ are in one-to-one correspondence with convex triangulations of $Q$. Such a correspondence may be described as follows. Let $\tau_{Q}$ be any triangulation of $Q$. It induces for $i=1,2$ a triangulation $\tau_{i}$ of $P_{i}$ and each four dimensional simplex of $\tau_{Q}$ is a join $\left\{e_{1}\right\} \times \sigma_{1} \star\left\{e_{2}\right\} \times \sigma_{2}$, where $\sigma_{i} \in \tau_{i}$. The corresponding three dimensional polytope in the decomposition of $P$ is then the Minkowsky sum $\sigma_{1}+\sigma_{2}$.

Take the joins $\left\{e_{1}\right\} \times P_{1} \star\left\{e_{2}\right\} \times\{(0,0,0)\},\left\{e_{1}\right\} \times\{(0,0, k)\} \star\left\{e_{2}\right\} \times P_{2}$, $\left\{e_{1}\right\} \times p_{1} \star\left\{e_{2}\right\} \times[(0,0,0),(2 k, 0,0)]$ and $\left\{e_{1}\right\} \times[(0,0, k),(0, k, 0)] \star\left\{e_{2}\right\} \times p_{2}$, and refine them in the unique possible way using convex triangulations $\tau_{1}$ and $\tau_{2}$. The resulting triangulation $\tau_{Q}$ of $Q$ is obviously convex and corresponds to the decomposition $\tau$ above. Therefore, by Lemma 3.1, the decomposition $\tau$ is a mixed decomposition.

To achieve the description of $\tau$, it remains to define triangulations $\tau_{1}$ and $\tau_{2}$. We simultaneously define associated distributions of signs $s_{1}, s_{2}^{c}$ and $s_{2}^{h}$. First, let us recall the notion of T-curves, which are, by definition, those curves constructed by means of the combinatorial patchworking (see [I1]). 
3.0.1. T-curves in $\mathbb{R} P^{2}$. For any positive integer number $m$, denote by $t_{m}$ the triangle with vertices $(0,0),(m, 0),(0, m)$ in $\mathbb{R}^{2}$. Start with a convex triangulation (with integral vertices) $\gamma$ of $t_{m}$ and a distribution of signs $s: \operatorname{vert}(\gamma) \rightarrow \pm 1$ at the vertices of $\gamma$. Extend $\gamma$ to a triangulation $\gamma^{*}$ of $t_{m}^{*}$ invariant with respect to $\mathcal{R}^{2}$. Extend $s$ to a distribution of signs on the set of vertices of $\gamma^{*}$ following the rule: $s(r(w))=s(w) \cdot(-1)^{\langle r, \bar{w}\rangle}$, where $\langle$,$\rangle is the usual scalar product in (\mathbb{Z} / 2)^{2}$. For each triangle of $\gamma^{*}$ having at least two vertices with different signs, select the segment joining the middle points of its two edges having endpoints with different signs. The union of the selected pieces is a piecewise linear curve in $t_{m}^{*}$, denote by $\tilde{a}$ its image in $\tilde{t}_{m}$.

Theorem 3.1 (Viro, see [I1]). There exists a nonsingular curve $A$ of degree $m$ in $\mathbb{R} P^{2}$ such that the pair $\left(\mathbb{R} P^{2}, \mathbb{R} A\right)$ is homeomorphic to $\left(\tilde{t}_{m}, \tilde{a}\right)$.

Such a curve $A$ is called a T-curve and may be obtained as the zero set of some T-polynomial associated with the considered triangulation and distribution of signs.

3.0.2. Triangulation and distribution of signs for $P_{1}$. Identify $p_{1}$ with the triangle $t_{k}$ in $\mathbb{R}^{2}$ using the map $(x, y, z) \rightarrow(y, z)$. We use a triangulation of $t_{k}$ and a distribution of signs at its vertices which produce a T-curve of degree $k$ in $\mathbb{R} P^{2}$ being an $M$-curve, i.e. having $\frac{(k-1)(k-2)}{2}+1$ ovals (see [I1]). Namely, take any primitive convex triangulation of $t_{k}$ (a triangulation of $t_{m}$ is called primitive if its set of vertices coincides with that of all the integer points contained in $t_{m}$ ) and the distribution of signs at its vertices which takes values +1 in any point with both coordinates even and -1 otherwise. Then, we extend the triangulation of $p_{1}$ to any convex triangulation $\tau_{1}$ of $P_{1}$, and choose any distribution of signs $s_{1}$ at the vertices of $\tau_{1}$ which extends the above distribution.

3.0.3. Triangulation and distributions of signs for $P_{2}$. Identify $p_{2}$ with the triangle $t_{2 k}$ using the map $(x, y, z) \rightarrow(x, y)$. Take any primitive convex triangulation $\gamma$ of $t_{2 k}$ and consider any distribution of signs $s_{\gamma}$ at its vertices which produce a T-curve of degree $2 k$ in $\mathbb{R} P^{2}$ whose real point set consists of $2 k^{2}+O(k)$ ovals such that $\frac{5 k^{2}}{3}+O(k)$ of them are even ovals (an oval of a curve in $\mathbb{R} P^{2}$ is called even, resp. odd, if it is lying inside an even, resp. odd, number of ovals of the curve). Such a T-curve does exist [Ha]. We define the restrictions of $s_{2}^{c}$ and $s_{2}^{h}$ to the set of vertices of $\gamma$ in the following way. On the subset of those vertices lying on the segment $[(0,0),(2 k, 0)]$, let $s_{2}^{c}$ and $s_{2}^{h}$ take value +1 in the points with even $x$-coordinate and -1 in the other points. On the complementary subset, let $s_{2}^{c}$ coincide with $s_{\gamma}$ and $s_{2}^{h}$ coincide with the opposite distribution $-s_{\gamma}$. Finally, take the cones with vertex $(0,0,2 k)$ over all the triangles of $\gamma$, and choose any sign for the vertex $(0,0,2 k)$. Consider two T-curves $A^{c}$ and $A^{h}$ of degree $2 k$ in $\mathbb{R} P^{2}$ associated with $\left(\gamma, s_{2}^{c}\right)$ and $\left(\gamma, s_{2}^{h}\right)$, respectively (in fact the restrictions of $s_{2}^{c}$ and $s_{2}^{h}$ to the set of vertices of $\left.\gamma\right)$. Both curves have $2 k^{2}+O(k)$ ovals in $\mathbb{R} P^{2}$ such that $\frac{5 k^{2}}{3}+O(k)$ of them are even ovals. Let $\tilde{a}^{c}$ and $\tilde{a}^{h}$ be the corresponding piecewise 
linear curves constructed in $\tilde{t}_{2 k}$. Replacing $s_{\gamma}$ by $-s_{\gamma}$ if necessary, assume that the ovals of $\tilde{a}^{c}$ bording from the exterior components of $\tilde{t}_{2 k} \backslash \tilde{a}^{c}$ on which $s_{2}^{c}$ takes values +1 are in one to one correspondence with the even ovals of $A^{c}$. Then, the ovals of $\tilde{a}^{h}$ bording from the exterior components of $\tilde{t}_{k-2} \backslash \tilde{a}^{h}$ on which $s_{2}^{h}$ takes values +1 are in one to one correspondence with the odd ovals of $A^{h}$.

Let $\widehat{X}_{k}, X_{2 k}^{c}$ and $X_{2 k}^{h}$ be surfaces in $\mathbb{C} P^{3}$ defined by T-polynomials $f_{1, t_{0}}, f_{2, t_{0}}^{c}$ and $f_{2, t_{0}}^{h}$ associated, respectively, with the pairs $\left(\tau_{1}, s_{1}\right),\left(\tau_{2}, s_{2}^{c}\right)$ and $\left(\tau_{2}, s_{2}^{h}\right)$ described above. Assume that $t_{0}$ is sufficiently small in order to have the properties stated in Proposition 2.2 for the pairs of surfaces $\left(\widehat{X}_{k}, X_{2 k}^{c}\right)$ and $\left(\widehat{X}_{k}, X_{2 k}^{c}\right)$. Let $X_{k}$ be a surface in $\mathbb{C} P^{3}$ obtained starting from $\widehat{X}_{k}$ as in 2.3 . It is defined by some Viro polynomial $p_{t}$. Denote by $A^{c}$ and $A^{h}$ the real curves given by the intersection of $X_{k}$ with $X_{2 k}^{c}$ and $X_{2 k}^{h}$, respectively.

Proposition 3.1. For sufficiently small $t>0$, the curves $\mathbb{R} A^{c}$ and $\mathbb{R} A^{h}$ are nonsingular curves with $3 k^{3}+O\left(k^{2}\right)$ connected components.

Proof. The proof is the same for both curves, let us only deal with the curve $A^{c}$. For sufficiently small $t>0$, the curve $\mathbb{R} A^{c}$ is nonsingular since it is a transversal intersection of two nonsingular surfaces. The polynomial $f_{2, t_{0}}^{c}$ does not vanish at $O$, hence there exists a neighbourhood $\mathcal{U}$ of $O$ in $\mathbb{R} P^{3}$ such that $\mathbb{R} A^{c} \subset \mathbb{R} P^{3} \backslash \mathcal{U}$. Then, by Proposition 2.4, the curve $\mathbb{R} A^{c}$ is homeomorphic to $\tilde{c}=\tilde{S}^{1} \cap \tilde{S}^{2, c}$, where $\tilde{S}^{1}$ and $\tilde{S}^{2, c}$ are surfaces in $\tilde{P}$ constructed as in 2.2 starting from $\left(\tau_{1}, s_{1}\right)$ and $\left(\tau_{2}, s_{2}^{c}\right)$, respectively. Denote by $\tilde{S}_{1} \subset \tilde{P}_{1}$ and $\tilde{S}_{2, c} \subset \tilde{P}_{2}$ the piecewise linear surfaces constructed as in 2.2 from $\left(\tau_{1}, s_{1}\right)$ and $\left(\tau_{2}, s_{2}^{c}\right)$, respectively.

The edge $[(0,0,0),(2 k, 0,0)]$ of $P_{2}$ is triangulated in $2 k$ edges. All the symmetric copies contained in $\{x \geq 0\}$ of these $2 k$ edges have endpoints with different signs, in other words, each of these copies is intersected by the surface $\tilde{S}_{2, c}$. Therefore, in each of the four orthants forming $\{x \geq 0\}$, the corresponding copy of $\Lambda_{1}$ contains $2 k$ pieces of $\tilde{S}^{2, c}$ and each piece is a triangle obtained as the Minkowsky sum of a copy of $p_{1}$ with the middle point of a copy of an edge lying on $[(0,0,0),(2 k, 0,0)]$. The map $(x, y, z) \rightarrow(y, z)$ allows us to identify the triangulation, and the associated distribution of signs, of each copy of $p_{1}$ contained in $\{x \geq 0\}$ with those obtained as in 3.0.1 for the corresponding copy of $t_{k}$. We have chosen a triangulation of $t_{k}$ and a distribution of signs at its set of vertices which produce a T-curve of degree $k$ in $\mathbb{R} P^{2}$ having $\frac{k^{2}}{2}+O(k)$ ovals. This means that the surface $\tilde{S}_{1}$ intersects the copies of $p_{1}$ contained in $\{x \geq 0\}$ in $\frac{k^{2}}{2}+O(k)$ ovals. Therefore, in $\{x \geq 0\}$, the symmetric copies of $\Lambda_{1}$ contain $\frac{k^{2}}{2}+O(k)$ pieces of $\tilde{S}^{1}$ and each piece is a cylinder obtained as the Minkowsky sum of one of these ovals with a copy of $[(0,0,0),(2 k, 0,0)]$. Finally, each of these cylinders intersects $2 k$ triangles of $\tilde{S}^{2, c}$, hence we obtain $k^{3}+O\left(k^{2}\right)$ ovals of $\tilde{c}$ contained in the symmetric copies of $\Lambda_{1}$.

The edge $[(0,0, k),(0, k, 0)]$ of $P_{1}$ is triangulated into $k$ edges. All the symmetric copies, contained in the four orthants forming $\{y \cdot z \geq 0\}$, of these $k$ edges are intersected by the surface $\tilde{S}_{1}$. Therefore, in each of these four orthants, the 
corresponding copy of $\Lambda_{2}$ contains $k$ pieces of $\tilde{S}^{1}$ and each piece is a triangle obtained as the Minkowsky sum of a copy of $p_{2}$ with the middle point of a copy of an edge lying on $[(0,0, k),(0, k, 0)]$. The map $(x, y, z) \rightarrow(x, y)$ allows us to identify the triangulation, and the associated distribution of signs, of each copy of $p_{2}$ contained in $\{y \cdot z \geq 0\}$ with those obtained as in 3.0.1 for the corresponding copy of $t_{2 k}$. We have chosen a triangulation of $t_{2 k}$ and a distribution of signs at its set of vertices which produce a T-curve of degree $2 k$ in $\mathbb{R} P^{2}$ having $2 k^{2}+O(k)$ ovals. This means that the surface $\tilde{S}_{2, c}$ intersects the copies of $p_{2}$ contained in $\{y \cdot z \geq 0\}$ in $2 k^{2}+O(k)$ ovals. Therefore, in $\{y \cdot z \geq 0\}$, the symmetric copies of $\Lambda_{2}$ contain $2 k^{2}+O(k)$ pieces of $\tilde{S}^{2, c}$ and each piece is a cylinder obtained as the Minkowsky sum of one of these ovals with a copy of $[(0,0,0),(2 k, 0,0)]$. Each of these cylinders intersects $k$ triangles of $\tilde{S}^{1}$, hence we obtain $2 k^{3}+O\left(k^{2}\right)$ ovals of $\tilde{c}$ contained in the symmetric copies of $\Lambda_{2}$.

Consequently the curve $\mathbb{R} A^{c}$ has at least $3 k^{3}+O\left(k^{2}\right)$ ovals. It is not difficult to see that $\mathbb{R} A^{c}$ has asymptotically no more connected components. Anyway, this follows from Harnack theorem since the genus of $A^{c}$ is $3 k^{3}+O\left(k^{2}\right)$.

Proposition 3.2. For sufficiently small $t>0$, the real point sets of the surfaces $Y_{m}^{c}$ and $Y_{m}^{h}$ of type $\mathcal{D}_{m}$ associated with $\left(X_{2 k}^{c}, X_{k}\right)$ and $\left(X_{2 k}^{h}, X_{k}\right)(m=2 k)$, respectively, are nonsingular surfaces. Moreover, if $b_{0}\left(\mathbb{R} V_{k-2}\right)=\alpha k^{3}+O\left(k^{2}\right)$ and $b_{1}\left(\mathbb{R} V_{k-2}\right)=\beta k^{3}+O\left(k^{2}\right)$, then one has

- $b_{0}\left(\mathbb{R} Y_{m}^{c}\right)=\frac{13+12 \alpha}{48} \cdot m^{3}+O\left(m^{2}\right), b_{1}\left(\mathbb{R} Y_{m}^{c}\right)=\frac{5+6 \beta}{24} \cdot m^{3}+O\left(m^{2}\right)$,

- $b_{0}\left(\mathbb{R} Y_{m}^{h}\right)=\frac{5+12 \alpha}{48} \cdot m^{3}+O\left(m^{2}\right), b_{1}\left(\mathbb{R} Y_{m}^{h}\right)=\frac{13+6 \beta}{24} \cdot m^{3}+O\left(m^{2}\right)$.

Proof. We have already seen in 2.4 that $\mathbb{R} Y_{m}^{c}$ and $\mathbb{R} Y_{m}^{h}$ are nonsingular surfaces for sufficiently small $t>0$. Set $\mathbb{R} X_{+}^{c}=\left\{Z \in \mathbb{R} X_{k}, f_{2, t_{0}}^{c}(Z) \geq 0\right\}$ and $\mathbb{R} X_{+}^{h}=\{Z \in$ $\left.\mathbb{R} X_{k}, f_{2 k, t_{0}}^{h}(Z) \geq 0\right\}$. Let $\mathcal{U}$ be any neighbourhood of $O$ in $\mathbb{R} P^{3}$ such that $\mathbb{R} X_{2 k}^{c} \cap$ $\mathcal{U}=\emptyset$ (resp. $\left.\mathbb{R} X_{2 k}^{c} \cap \mathcal{U}=\emptyset\right)$. By Propositions 2.3 and 2.4, for sufficiently small $t>0$, connected components of $\mathbb{R} X_{k}$ contained in $\mathcal{U}$ are connected components of $\mathbb{R} X_{+}^{c}$ (resp. $\mathbb{R} X_{+}^{h}$ ) and the topology of $\mathbb{R} X_{k} \cap \mathcal{U}$ coincides with that of some affine part of $\mathbb{R} V_{k-2}$. It follows (see Proposition 2.3) that the Betti numbers $b_{0}$ and $b_{1}$ of the largest subset of $\mathbb{R} X_{+}^{c}$ (resp. $\mathbb{R} X_{+}^{h}$ ) consisting of closed (i.e. without boundary) connected components verify $b_{0}=\alpha k^{3}+O\left(k^{2}\right)$ and $b_{1}=\beta k^{3}+O\left(k^{2}\right)$.

Let $\tilde{S}^{1}, \tilde{S}^{2, c}$ and $\tilde{S}^{2, h}$ be surfaces constructed in $\tilde{P}$ starting from $\left(\tau_{1}, s_{1}\right),\left(\tau_{2}, s_{2}^{c}\right)$ and $\left(\tau_{2}, s_{2}^{h}\right)$, respectively. Denote by $\tilde{S}_{+}^{c}$ (resp. $\tilde{S}_{+}^{h}$ ) the intersection of $\tilde{S}^{1}$ with connected components of the complementary part of $\tilde{S}^{2, c}\left(\operatorname{resp} . \tilde{S}^{2, h}\right)$ in $\tilde{P} \backslash \mathcal{V}$ on which $s_{2}^{c}$ (resp. $s_{2}^{h}$ ) takes the value +1 . In $\{x \geq 0\}$, each cylinder of $\tilde{S}^{1}$ (see the proof of Proposition 3.1) contained in a symmetric copy of $\Lambda_{1}$ is cut by $\tilde{S}^{2, c}$ (resp. $\left.\tilde{S}^{2, h}\right)$ into $2 k+O(1)$ small cylinders and two non consecutive of them are connected components of $\tilde{S}_{+}^{c}\left(\operatorname{resp} . \tilde{S}_{+}^{h}\right)$. There are, in $\{x \geq 0\}, \frac{k^{2}}{2}+O(k)$ cylinders of $\tilde{S}^{1}$ contained in the symmetric copies of $\Lambda_{1}$, hence we obtain $\frac{k^{3}}{2}+O\left(k^{2}\right)$ connected components of $\tilde{S}_{+}^{c}$ (resp. $\tilde{S}_{+}^{h}$ ), and thus of $\mathbb{R} X_{+}^{c}$ (resp. $\mathbb{R} X_{+}^{h}$ ) by Proposition 2.4, which are homeomorphic to a cylinder. As in 3.0.1, let $\tilde{a}^{c}$ and $\tilde{a}^{h}$ be the piecewise 
linear curves constructed in $\tilde{t}_{2 k}$ starting from $\left(\gamma, s_{2}^{c}\right)$ and $\left(\gamma, s_{2}^{h}\right)$, respectively. In $\{y \cdot z \geq 0\}$, each symmetric copy of $\Lambda_{2}$ contains $k$ triangles of $\tilde{S}^{1}$ and each oval of $\tilde{a}^{c}$ (resp. $\tilde{a}^{h}$ ) lying on a symmetric copy of $t_{2 k}$ gives rise to $k$ ovals of the curve $\tilde{S}^{1} \cap \tilde{S}^{2, c}$ (resp. $\tilde{S}^{1} \cap \tilde{S}^{2, h}$ ) lying on the corresponding $k$ triangles of $\tilde{S}^{1}$ (see the proof of Proposition 3.1). Each connected component of $\tilde{t}_{2 k} \backslash \tilde{a}^{c}$ (resp. $\left.\tilde{t}_{2 k} \backslash \tilde{a}^{h}\right)$ on which $s_{2}^{c}$ (resp. $s_{2}^{h}$ ) takes the value +1 and lying on a symmetric copy of $t_{2 k}$ gives rise to $k$ homeomorphic connected components of $\tilde{S}_{+}^{c}\left(\right.$ resp. $\tilde{S}_{+}^{h}$ ) lying on the corresponding $k$ triangles of $\tilde{S}^{1}$. The connected components of $\tilde{t}_{2 k} \backslash \tilde{a}^{c}$ on which $s_{2}^{c}$ takes the value +1 are borded from the exterior by even ovals of $\tilde{a}^{c}$ and from the interior by odd ovals of $\tilde{a}^{c}$. Therefore, we obtain $\frac{5 k^{3}}{3}+O\left(k^{2}\right)$ connected components of $\tilde{S}_{+}^{c}$, and thus of $\mathbb{R} X_{+}^{c}$, which are homeomorphic to disks with holes. The total number of these holes is $\frac{k^{3}}{3}+O\left(k^{2}\right)$. The connected components $\tilde{t}_{2 k} \backslash \tilde{a}^{h}$ on which $s_{2}^{h}$ takes the value +1 are borded from the exterior by odd ovals of $\tilde{a}^{h}$ and from the interior by even ovals of $\tilde{a}^{h}$. Therefore, we obtain $\frac{k^{3}}{3}+O\left(k^{2}\right)$ connected components of $\tilde{S}_{+}^{h}$, and thus of $\mathbb{R} X_{+}^{h}$, which are homeomorphic to disks with holes. The total number of these holes is $\frac{5 k^{3}}{3}+O\left(k^{2}\right)$. It is not difficult to see that $\mathbb{R} X_{+}^{c}$ (resp. $\mathbb{R} X_{+}^{h}$ ) has asymptotically no more connected components (this follows from Proposition 3.1). The computation of the asymptotic behaviour of the Betti numbers of $\mathbb{R} Y_{m}^{c}$ and $\mathbb{R} Y_{m}^{h}$ is now obvious.

\section{Iteration and proof of Theorem 1.1}

\subsection{Iteration}

We construct, by induction on the integer number $n \geq 0$, two families $C^{n}=$ $\left\{C_{m}^{n}, m>m_{n}\right\}$ and $H^{n}=\left\{H_{m}^{n}, m>m_{n}\right\}$ consisting of nonsingular real surfaces $C_{m}^{n}$ and $H_{m}^{n}$ of degree $m$ in $\mathbb{C} P^{3}$ for any integer number $m>m_{n}$.

As starting families $C^{0}$ and $H^{0}$, let us take a family of surfaces $X_{m}$ of degree $m$ in $\mathbb{C} P^{3}$ constructed by Viro [V1] which verify $b_{0}\left(\mathbb{R} X_{m}\right)=\frac{m^{3}}{6}+O\left(m^{2}\right)$ and $b_{1}\left(\mathbb{R} X_{m}\right)=\frac{2 m^{3}}{3}+O\left(m^{2}\right)$. We describe now the induction step. Assume that the families $C^{n}$ and $H^{n}$ have been constructed. For each integer number $k$ such that $k-2>m_{n}$, we construct the surfaces $C_{2 k}^{n+1}$ and $H_{2 k}^{n+1}$ of the families $C^{n+1}$ and $H^{n+1}$ as follows. As in 2.3, use the surfaces $C_{k-2}^{n}$ and $H_{k-2}^{n}$ in place of the surface $V_{k-2}$ in order to perform two smoothings of the surface $\widehat{X}_{k}$ constructed in Section 3 (perturbing $C_{k-2}^{n}$ and $H_{k-2}^{n}$ a little if necessary, one can assume that these surfaces are defined by nondegenerate polynomials with Newton polytope $T_{k-2}$ ). Let $X_{k}^{c}$ and $X_{k}^{h}$ be the resulting surfaces, respectively. Consider now surfaces $Y_{2 k}^{c}$ and $Y_{2 k}^{h}$ of type $\mathcal{D}_{2 k}$ associated with $\left(X_{2 k}^{c}, X_{k}^{c}\right)$ and $\left(X_{2 k}^{h}, X_{k}^{h}\right)$, respectively, where $X_{2 k}^{c}$ and $X_{2 k}^{h}$ are the surfaces constructed in Section 3. Assume that the parameters of Viro's polynomials defining $X_{k}^{c}$ and $X_{k}^{h}$ are sufficiently small in order to have the properties described in Proposition 3.2. The surfaces $C_{2 k}^{n+1}$ and $H_{2 k}^{n+1}$ are 
then obtained applying to $Y_{2 k}^{c}$ and $Y_{2 k}^{h}$, respectively, the equivariant deformation described in 2.1.

At this point of the construction, the family $C^{n+1}$ (resp. $H^{n+1}$ ) contains surfaces $C_{2 k}^{n+1}$ (resp. $H_{2 k}^{n+1}$ ) for any integer number $k$ such that $k-2>m_{n}$. Take the union of each surface $C_{2 k}^{n+1}$ (resp. $H_{2 k}^{n+1}$ ) with a plane and smooth the resulting surface in order to obtain the whole families $C^{n+1}$ and $H^{n+1}$ with $m_{n+1}=2\left(m_{n}+2\right)$. The following result may be easily derived from Proposition 3.2.

Proposition 4.1. The surfaces $C_{m}^{n}$ and $H_{m}^{n}$ verify

(1) $b_{0}\left(\mathbb{R} C_{m}^{n}\right) \sim \alpha_{c}^{n} \cdot m^{3}$ and $b_{1}\left(\mathbb{R} C_{m}^{n}\right) \sim \beta_{c}^{n} \cdot m^{3}$ when $m \rightarrow+\infty$, where $\alpha_{c}^{n}$ and $\beta_{c}^{n}$ are recursively defined by

$$
\alpha_{c}^{0}=\frac{1}{6}, \alpha_{c}^{n+1}=\frac{13+12 \alpha_{c}^{n}}{48} \quad \text { and } \quad \beta_{c}^{0}=\frac{2}{3}, \beta_{c}^{n+1}=\frac{5+6 \beta_{c}^{n}}{24},
$$

(2) $b_{0}\left(\mathbb{R} H_{m}^{n}\right) \sim \alpha_{h}^{n} \cdot m^{3}$ and $b_{1}\left(\mathbb{R} H_{m}^{n}\right) \sim \beta_{h}^{n} \cdot m^{3}$ when $m \rightarrow+\infty$, where $\alpha_{h}^{n}$ and $\beta_{h}^{n}$ are recursively defined by

$$
\alpha_{h}^{0}=\frac{1}{6}, \alpha_{h}^{n+1}=\frac{5+12 \alpha_{c}^{n}}{48} \quad \text { and } \quad \beta_{h}^{0}=\frac{2}{3}, \beta_{h}^{n+1}=\frac{13+6 \beta_{h}^{n}}{24} .
$$

\subsection{Proof of Theorem 1.1}

The sequences $\alpha_{c}^{n}$ and $\beta_{h}^{n}$ defined in Proposition 4.1 converge to $\frac{13}{36}$ and $\frac{13}{18}$, respectively. Therefore, for sufficiently large $n$ and $m$, we have

$$
b_{0}\left(\mathbb{R} C_{m}^{n}\right)>\left(\frac{13}{36}-\epsilon\right) \cdot m^{3} \quad \text { and } \quad b_{1}\left(\mathbb{R} H_{m}^{n}\right)>\left(\frac{13}{18}-\epsilon\right) \cdot m^{3}
$$

for a given $\epsilon>0$.

\section{Concluding remarks}

1. Viro's conjecture. Viro proposed the inequality $b_{1}(\mathbb{R} X) \leq h^{1,1}(X)$ for any real nonsingular projective and simply connected surface $X$ as a natural generalization of the Ragsdale conjecture for real plane curves. Since then, both conjectures were shown to be false in general (see [I1, I2, B2, B1]). However, both remain open if the surface (resp. the curve) is assumed to be an $M$-surface (resp. an $M$-curve) i.e. if it is maximal with respect to the Smith-Thom inequality (resp. Harnack inequality). For a surface $X_{m}$ in $\mathbb{C} P^{3}$, Viro's conjecture asserts that $b_{1}(\mathbb{R} X) \leq \frac{2}{3} m^{3}+-2 m^{2}+\frac{7}{3} m$. Furthermore, $X_{m}$ is an $M$-surface if $b_{*}\left(\mathbb{R} X_{m}\right)=2 b_{0}\left(\mathbb{R} X_{m}\right)+b_{1}\left(\mathbb{R} X_{m}\right)=m^{3}-4 m^{2}+6 m$. We note that the surfaces $H_{m}^{n}$ constructed in this paper are not far from being $M$-surfaces since $b_{*}\left(\mathbb{R} H_{m}^{n}\right)=m^{3}+O\left(m^{2}\right)$ (this follows from $2 \alpha_{h}^{n}+\beta_{h}^{n}=1$, which is easily proven 
recursively) and are at the same time (for large $m$ and $n$ ) strong counter-examples to the Viro conjecture.

2. Limits of the method. There is an increasing function relating the coefficients $\frac{13}{36}$ and $\frac{13}{18}$ in Theorem 1.2 with the coefficient $\frac{5}{3}$ in the number $\frac{5}{3} \cdot k^{2}+O(k)$ of even ovals of the plane curves we have used. Namely, using curves with $a \cdot k^{2}+O(k)$ even ovals, one obtains coefficients $\frac{a}{6}+\frac{1}{12}$ and $\frac{a}{3}+\frac{1}{6}$ instead of $\frac{13}{36}$ and $\frac{13}{18}$ in Theorem 1.2, respectively. The inequality $a \leq \frac{7}{4}$ is well-known in the topology of real algebraic curves. This implies that our construction cannot give lower bounds better than $\frac{3}{8}$ and $\frac{3}{4}$ for $l_{0}$ and $l_{1}$, respectively.

3. Generalization. Our construction can be generalized in order to construct real nonsingular algebraic hypersurfaces in $\mathbb{C} P^{n}$ with large Betti numbers for any integer number $n \geq 1$. In this way, we obtain increasing functions relating the real numbers $l_{i}^{(n)}$ introduced in Remark 1.1 with corresponding real numbers for lower dimensional real hypersurfaces. All this will appear in a forthcoming paper.

\section{References}

[B1] F. Bihan, Constructions Combinatoires de Surfaces Algébriques Réelles, Thesis, University of Rennes (France), 1998.

[B2] F. Bihan, Betti numbers of real numerical quintic surfaces, in: Topology, Ergodic Theory, Real Algebraic Geometry. Rokhlin's Memorial, 31-39, ed. V. Turaev and A. Vershik, Amer. Math. Soc. Transl. ser. 2, vol. 202, 2001.

[D-K] A. Degtyarev and V. Kharlamov, Topological properties of real algebraic varieties: du côté de chez Rokhlin, Russian Mathematical Surveys, Vol. 55, No 4, 2000.

[Ha] B. Haas, Les multilucarnes: nouveaux contre-exemples a la conjecture de Ragsdale, C.R. Acad. Sci. Paris. Ser. I. (1995), 1507-1512.

[Ho] E. Horikawa, Deformations of Sextic Surfaces, Topology 32, no. 4 (1993), 757-772.

[I1] I. Itenberg, Counter-examples to Ragsdale Conjecture and T-curves, Cont. Math. (Proceedings, Michigan 1993) 182 (1995), 55-72.

[I2] I. Itenberg, Topology of real algebraic T-surfaces, Revista Matematica 10 (1997), Special Issue, suppl., 131-152.

[I-K1] I. Itenberg and V. M. Kharlamov, Towards the maximal number of components of a nonsingular surface of degree 5 in $\mathbb{R} P^{3}$, Amer. Math. Soc. Trans. (2) 173 (1996), 111-118.

[I-K2] I. Itenberg and V. M. Kharlamov, Private communication.

[K1] V. M. Kharlamov, Topological types of nonsingular surfaces in $\mathbb{R} P^{3}$ of degree 4, Funct. Anal. Appl. 10 (1976), 55-68.

[K2] V. M. Kharlamov, Real algebraic surfaces, Proc. Internat. Congress Math., Helsinki 1 (1978), 421-428 (in Russian).

[O] S. Orevkov, Real quintic surface with 23 components, C. R. Acad. Sci. Paris Ser. 1 Math. 333 (2001), no. 2, 115-118.

[R] J.-J. Risler, Construction d'hypersurfaces réelles [d'apres Viro], Séminaire Bourbaki vol. 1992-93, Astérisque 216 (1993), Exp. no. 763, 3, 69-86.

[S1] B. Sturmfels, Viro's theorem for complete intersections, Annali della Scuola Normale Superiore di Pisa (4) 21 (1994), no. 3, 377-386.

[S2] B. Sturmfels, On the Newton polytope of the resultant, Journal of Algebraic Combinatorics 3 (1994), 207-236. 
[V1] O. Viro, Construction of M-surfaces, Funktsional. Anal. i Prilozhen. 13 (1979), no. 3, $71-72$.

[V2] O.Viro, Construction of multicomponent real algebraic surfaces, Sov. Math. Doklady 20 (1979), 991-995.

[V3] O. Viro, Gluing of algebraic hypersurfaces, smoothing of singularities and contruction of curves, in: Proc. Leningrad Int. Topological Conf. (Leningrad, Aug. 1983), 149-197 (in Russian), Nauka, Leningrad, 1983.

[V4] O. Viro, Gluing of plane algebraic curves and construction of curves of degree 6 and 7 , 187-200, Lect. Notes Math., vol 1060, Springer-Verlag, Berlin-Heidelberg, 1984.

[V5] O. Viro, Real algebraic plane curves: constructions with controlled topology, Leningrad Math. J. 1 (1990), 1059-1134.

[W] G. Wilson, Hilbert's sixteen problem, Topology 17 (1978), no. 1, 53-74.

\section{F. Bihan}

Université de Lausanne

Faculté des Sciences

Institut de Mathématiques (IMA)

$\mathrm{BCH}$

CH-1015 Lausanne

Switzerland

e-mail: Frederic.Bihan@ima.unil.ch

(Received: April 26, 2000) 